\title{
Factores generadores de éxito para la gestión del conocimiento mediante la aplicación de un modelo de ecuaciones estructurales
}

\author{
ROSA MARÍA ROMERO GONZÁLEZ* \\ Universidad Autónoma de Querétaro (UAQ) - México \\ Recibido el 31-05-17; primera evaluación el 29-01-19; \\ segunda evaluación el 20-06-19; tercera evaluación \\ el 21-06-19; aceptado 21-08-19.
}

\section{RESUMEN}

El objetivo fue analizar cómo se gestiona el conocimiento en una institución de educación superior, a través del análisis un modelo de ecuaciones estructurales. La investigación se realizó con base en el modelo propuesto por Molina y Marsal (2002), analizando la implicación de la alta dirección, cultura organizativa, capacidad de gestión, tecnología, procesos de gestión del conocimiento e indicadores. El cuestionario consistió de 34 preguntas en escala Likert. El coeficiente Alfa de Cronbach obtenido para las seis variables fue 0.948 . Se observó que los directivos se integran y apoyan la gestión del conocimiento, que los participantes se consideran responsables de su propia información. A través del análisis de un modelo de ecuaciones estructurales, se identificó la influencia de las variables observadas en la variable en estudio.

Palabras clave: ecuaciones estructurales, factores de éxito, gestión del conocimiento

\footnotetext{
* Doctora en Administración por la Universidad Autónoma de Querétaro (UAQ), DocenteInvestigador en la Facultad de Informática - UAQ. Miembro de los programas académicos del Doctorado en Tecnologías Educativa y Ciencias Económico Administrativas, y de Licenciatura en Ingeniería de Software, Ingeniería en Computación e Informática. Escritora de artículos sobre gestión del conocimiento y gestión de la tecnología. Correo Electrónico: rossyrg04@yahoo.com.mx
} 


\section{Successful generating factors for knowledge management through the application of a structural equation model}

\section{Abstract}

The purpose was to analyze how the transfer of knowledge is managed in an institution of higher education by analyzing a model of structural equations. The research was conducted on the basis proposed by Molina and Marsal (2002) model, analyzing the implication of the high direction, organizational culture, management skills, technology, knowledge management processes and indicators. The questionnaire consisted of 34 questions in Likert scale. Cronbach's alpha coefficient obtained for the six variables was .948. It was noted that managers integrate and support knowledge management; participants are considered responsible for their own information. The influence of the observed variables in the variable under study was identified through analysis of a structural equation model.

Keywords: Knowledge management, knowledge transfer, processes, successful factors

\section{Fatores geradores de sucesso para a gestáo do conhecimento por meio da aplicaçáo de um modelo de equaçóes estruturais}

\section{RESUMO}

O objetivo foi analisar como o conhecimento em uma instituição de ensino superior é gerido pela análise de um modelo de equaçóes estruturais. A pesquisa foi realizada com base proposto por Molina e Marsal (2002) modelo, analisando o envolvimento da alta administração, cultura organizacional, competências de gestáo, tecnologia, processos de gestão do conhecimento e indicadores. O questionário consistia em 34 perguntas em escala de Likert. Cronbach coeficiente alfa obtidos para os seis variáveis foi 0,948 . Observou-se que os gerentes de integrar e gestão de conhecimento de suporte, os participantes são considerados responsáveis por suas próprias informaçôes. Através da análise de uma equação estrutural modelar a influência das variáveis observadas na variável em estudo identificou.

Palavras-chave: Gestão do conhecimento, fatores de sucesso, processos, transmissão de conhecimento 


\section{INTRODUCCIÓN}

Las universidades están constituidas por docentes, alumnos y personal administrativo: Todos ellos pertenecen al grupo de trabajadores del conocimiento. En este trabajo, la investigación se centra en la forma en cómo los docentes transfieren el conocimiento y en cómo lo reciben los alumnos. Asimismo, se analiza el estilo de los directivos para integrarse en esta dinámica. Por lo tanto, el propósito de distinguir las técnicas para gestionar el conocimiento en la institución se originó debido a que se observó que hay escases de procedimientos para conservarlo, aunque existen esquemas universitarios que coadyuvan para su permanencia; dentro de la facultad, la información se encuentra dispersa, los docentes-investigadores se centran en sus proyectos de investigación generando productos y resultados muy valiosos que deberían ser compartidos y conocidos por toda la comunidad. Además de que el tiempo asignado a la docencia, a la investigación y para algunas actividades administrativas reduce tiempo establecido para convivir y compartir conocimientos valiosos que se generan a través de la experiencia. Debido a las limitaciones de tiempo y a que tienen múltiples actividades que atender, el espacio de tiempo para reflexionar o discutir sus experiencias es limitado; aunque los profesores se agrupen a través de cuerpos académicos y pasen tiempo juntos, se requiere de un espacio asignado específicamente para exponer sus conocimientos.

Por su parte, las tecnologías, al actuar como agentes inteligentes, ayudan a los docentes y a los alumnos a promover una participación activa y reflexiva en la construcción del aprendizaje, amplifican la experiencia social, proporcionan evidencias sobre el progreso de los estudiantes y estimulan su juicio crítico. Pero ¿cómo se puede estimular que los estudiantes realicen un análisis crítico del conocimiento que están recibiendo? Cabero, Ballesteros y López (2015) sugieren que, a través de mapas conceptuales interactivos, se faciliten la participación activa en la construcción de su propio conocimiento.

Por lo tanto, con la finalidad de vincular los modelos de ecuaciones estructurales (SEM) con los factores de éxito y con la gestión del conocimiento de una institución de educación superior, se ha desarrollado un modelo que presenta el impacto de las variables observadas en la variable latente que representa el objetivo principal de las Instituciones de Educación Superior para la generación y gestión del conocimiento (Secretaría de Educación Pública, 2015). 


\section{MarCo teórico}

\subsection{Antecedentes}

Para que la gestión del conocimiento aumente el capital intelectual en una organización, es importante administrar eficientemente el conocimiento de los docentes e investigadores de la institución para generar ventajas competitivas sostenibles en el tiempo. El reto es que el proceso de globalización es un motor de innovación que ha generado la competencia internacional en las instituciones educativas. De acuerdo con el Knowledge Master (2007), el rol de un administrador del conocimiento, ingeniero del conocimiento o trabajador del conocimiento es «identificar, recoger, sintetizar, organizar y administrar el conocimiento de la organización y de sus servicios informativos en apoyo de las unidades organizativas» (p. 1). Entonces surgen las preguntas en las instituciones de educación superior: ¿Cómo se recolecta el conocimiento?, ¿de qué manera se comprende y se valora en beneficio de la organización, de sus alumnos, docentes y empleados? Fernández (2015) considera que los profesionales del conocimiento deben tener un buen dominio y manejo de las herramientas tecnológicas que potencialicen la generación y producción del conocimiento requerido por las instituciones, con la finalidad de contribuir a crear ventajas competitivas. Con todo esto, se puede decir que las instituciones educativas deben tener estrategias que permitan incluir la constitución de bases del conocimiento, a través de las tecnologías de la información.

\subsection{Gestión del conocimiento}

Las redes internacionales de universidades promueven el fortalecimiento de los programas académicos, estableciendo estrategias que potencian la generación, transformación y gestión del conocimiento en cada una de las universidades; de igual forma, favorecen la consolidación de vínculos entre investigadores de distintos países y entre sus estudiantes. Normalmente, los investigadores sienten la necesidad de conocer los últimos avances científicos para aplicarlos al entorno que los rodea. El conocimiento es una capacidad humana adecuada para abstraer los conceptos de un conjunto de situaciones establecidas, se genera a través del razonamiento o la inferencia y se enriquece si este conocimiento está en transformación constante.

El término "gestión del conocimiento" se usa en un sentido genérico para incluir todo tipo de intercambio de conocimiento entre individuos, equipos, grupos y organizaciones, bien sea o no intencional, debe tener un propósito claro y definido. Argote (1999) señala que incluye la comunicación 
del conocimiento desde una fuente que representa a los docentes para que el conocimiento se aprenda y se aplique por parte de los alumnos, quienes representan la fuente receptora del conocimiento. En general, la fuente y el receptor pueden ser individuos, grupos, equipos, unidades organizacionales u organizaciones enteras en cualquier combinación. Ko, Kirsch y King (2005) han agregado factores de comunicación, porque consideran que son importantes para procesos de implementación de la gestión del conocimiento. Hartwick y Barki (2001) mencionan que se requieren muchas estrategias e interacciones para que el conocimiento sea totalmente gestionado. Es por esto que se debe motivar la relación entre los compañeros para que compartan sus experiencias y los conocimientos valiosos que poseen; $y$, de esta manera, impulsar la gestión del conocimiento en la organización. Para Herrera y Giraldo (2010), es importante adaptar el entorno de la institución, utilizando sus propios recursos para reforzar los conocimientos existentes y generar nuevos conocimientos.

\subsection{Factores de éxito}

Para que la gestión del conocimiento sea próspera, Digman (1990) indica que se debe dar especial interés y una continua atención a los factores de éxito para ser reflejados en los resultados. Estos factores son críticos, se pueden controlar y actúan como facilitadores. Molina y Marsal (2002), y De Freitas y Yaber (2015) señalan que los factores de éxito pueden ser apoyo de la alta gerencia, involucramiento de la alta gerencia, gestión de recursos humanos, habilidades, liderazgo, tamaño de la empresa, estructura organizativa, cultura organizacional, motivación, grupos de soporte para la gestión del conocimiento, métricas, sistema de recompensas, infraestructura tecnológica, asignación de recursos, plan eficaz de gestión del conocimiento, colaboración, objetivos definidos y benchmarking. Por su parte, López, Marulanda y López (2015) consideran el ciclo de vida, las tecnologías, la cultura organizacional, los procesos estratégicos, procesos misionales, procesos de apoyo, competencias personales y relaciones sociales.

\subsection{Modelos de ecuaciones estructurales}

Los modelos de ecuaciones estructurales (SEM) son una herramienta estadística para probar fundamentos teóricos valorando la relación entre constructos y permiten la estimación de ecuaciones de regresión múltiples de manera simultánea. Con este paradigma es posible establecer modelos que permiten analizar la complejidad del comportamiento humano a través de relaciones. 
Kline (2011) señala que los resultados dependen de la calidad de las hipótesis en las que se basa el análisis. Considera que es imposible analizar un modelo con variables latentes que representan construcciones hipotéticas sin pensar en cómo medir los constructos. Para comprenderlos, es importante entender los principios de correlación/regresión múltiple, la interpretación de los resultados de las pruebas estadísticas y las técnicas de detección de datos. En una investigación es importante evaluar las hipótesis, explicar cualquier hallazgo inesperado, relacionar los resultados con los estudios previos y reflexionar sobre las implicaciones en investigaciones futuras.

Para Schumacker y Lomax (2010), el objetivo del análisis SEM es determinar el grado en que los datos de las muestras apoyan un modelo teórico, llegando a ser hipótesis. Si los datos de la muestra no son compatibles con el modelo teórico, entonces se puede modificar y volver a probar el modelo original. Enfatizan que se ponen a prueba los modelos teóricos para analizar las relaciones complejas entre los constructos a través de las hipótesis.

Los modelos de ecuaciones estructurales (SEM) permiten medir y observar el comportamiento de las variables observadas y las variables que no se pueden observar. Para Byrne (2010), un modelo de SEM es una metodología estadística que utiliza un enfoque confirmatorio (prueba de hipótesis) del análisis de múltiples variables, aplicado a una teoría estructural relacionada con un fenómeno determinado. Para Gutiérrez-Doña (2008), son «ecuaciones lineales de matrices, en las cuales se pueden estimar los parámetros desconocidos a partir de los datos observados» (p. 11). De acuerdo con Sik-Yum (2007), es un modelo de regresión con variables latentes. Mientras que para Ullman (2006) es una "colección de técnicas estadísticas entre una o más variables independientes (ya sean continuas o discretas) y una o más variables dependientes (ya sean continuas o discretas)» (p. 37). Estas variables pueden ser medibles a través de observación directa o a través de variables latentes que no son observables directamente.

Manzano y Zamora (2009) exponen que en los modelos de ecuaciones estructurales hay tres tipos de parámetros: libres, fijos y de restricción. Los parámetros libres se estiman durante el proceso: las varianzas y covarianzas de las variables independientes, los coeficientes que conectan a las variables latentes con sus variables observadas, los que conectan a las variables latentes con latentes y los que conectan a variables observadas con observadas. A los parámetros fijos se les asigna de inicio un valor constante. Los parámetros de restricción expresan una hipótesis acerca de sus valores, por lo que se igualan a un valor particular, o se asume que son iguales a otros parámetros. Sik-Yum (2007) conceptualiza que los modelos SEM tienen dos componentes principales: 
el modelo de análisis confirmatorio que relaciona las variables latentes con todas sus variables observadas (indicadores) y toma en cuenta la variable error. Este modelo puede considerarse como un modelo de regresión que calcula el coeficiente de las variables observadas a través de un pequeño número de variables latentes. El segundo componente es una ecuación de regresión (ecuación estructural) que calcula el coeficiente de las variables latentes endógenas (dependiente) en términos de las variables latentes exógenas (independiente). Las variables latentes son al azar, pues no se pueden analizar por técnicas de regresión simples basadas en observación abiertas. Por su parte, Manzano y Zamora (2009) clasifican los diferentes modelos de ecuaciones estructurales como modelos recursivos, no recursivos, modelo factorial confirmatorio, modelo de regresión estructural, el modelo mimic y modelo de crecimiento.

Grace (2006) representa un modelo de ecuaciones estructurales (ver Figura 1) como dos o más ecuaciones estructurales para modelar la relación multivariante que tiene influencia y respuesta simultánea.

Figura 1. Representación gráfica de un modelo de ecuaciones estructurales

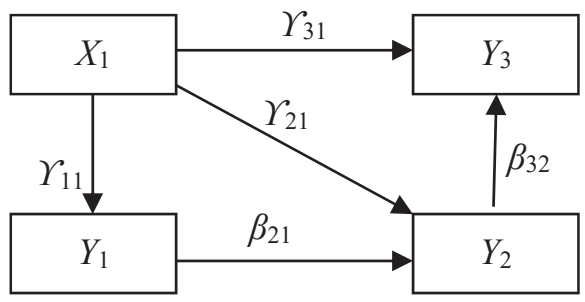

Fuente: Grace (2006, p. 11)

Esta representación gráfica se realiza a través del diagrama de trayectorias, en el cual las cajas o rectángulos representan las variables observadas, las flechas entre los rectángulos representan la relación directa, $\gamma$ representa el efecto de $x$ en la variable $y$, mientras que beta $(\beta)$ representa los efectos de las $y$ 's en las otras y's, alfa $(\alpha)$ simboliza la intersección de la población, las $\varsigma$ representan en términos de error para las variables de respuesta. Por tanto, este modelo de ecuaciones estructurales puede definirse como $Y_{1}=\alpha_{1}+\gamma_{11} X_{1}+\zeta_{1} ; Y_{2}=\alpha_{2}+$ $\beta_{21} Y_{1}+Y_{21} X_{1}+\varsigma_{2}$ y $Y_{3}=\alpha_{3}+\beta_{32} Y_{2}+\Upsilon_{31} X_{1}+\varsigma_{3}$. Partiendo de que la ecuación $Y_{1}=\alpha_{1}+Y_{11} X_{1}+\zeta_{1}$ representa el efecto que tiene la variable $x$ sobre $y$, se puede decir que es una ecuación estructural, solo si existe evidencia suficiente de que todas las fuentes disponibles permiten la interpretación del efecto causal que tiene $X_{1}$ sobre $Y_{1}$. Por lo tanto, si existe esta relación entonces el parámetro $\Upsilon_{11}$ proporciona una estimación de ese efecto. 
Los modelos incluyen un término de error estructural, distorsión, perturbación o de error ( $)$ para cada variable endógena. Gujarati y Porter (2009) la definen como una variable aleatoria (estocástica) con propiedades probabilísticas bien definidas. Este término de error tiene en cuenta todas las fuentes de variación que no están consideradas en las relaciones de regresión que predicen las variables endógenas. «La estimación consistente de los parámetros del modelo exige que estos términos de error no estén correlacionados con las variables exógenas» (p. 484).

\section{Metodología}

Partiendo de la pregunta central de investigación «¿Qué factores impulsan la gestión del conocimiento en una Institución de Educación Superior?», se consideró la hipótesis como «si la institución de educación superior en el estado de Querétaro conoce los factores de éxito para la gestión del conocimiento, entonces se impulsará la gestión del conocimiento». Por tanto, el objetivo central es identificar los factores que impulsan la gestión del conocimiento a partir un modelo propuesto por Molina y Marsal (2002). Se realizó un análisis de los parámetros importantes para la gestión del conocimiento a través de la aplicación de 135 encuestas con carácter anónimo en la Facultad de Informática de la Universidad Autónoma de Querétaro. El cuestionario consistió de 34 preguntas con base en una escala Likert. Las variables fueron implicación de la alta dirección, cultura organizativa, capacidad de gestión, tecnología, procesos de gestión del conocimiento e indicadores. La encuesta se aplicó a personas del género femenino $(50.4 \%)$ y masculino $(49.6 \%)$ con puestos directivos $(5.9 \%)$, docentes $(40.7 \%)$ y alumnos $(53.3 \%)$. La edad estuvo entre 15 a 20 ańos $(0.7 \%)$, de 21 a $25(11 \%)$, de 26 a 30 ańos (29.6\%), de 31 a 40 ańos (42.2\%), de 41 a 50 años (17\%) y de más de 51 ańos $(2.2 \%)$.

\subsection{Validez y confiabilidad}

Con los datos obtenidos, se realizó un análisis de fiabilidad mediante el cálculo del coeficiente alfa de Cronbach para cada variable. Para la variable «implicación de la alta dirección», se obtuvo una escala de fiabilidad de 0.842 , para «cultura organizativa» fue de 0.791 , en "capacidad de gestión» se obtuvo 0.920 , en «tecnología» fue 0.828 , en "procesos de gestión del conocimiento» se obtuvo 0.789 , finalmente, para «indicadores», la escala de fiabilidad fue de 0.851. En promedio, la fiabilidad media para las seis variables fue de 0.948 . 
En la prueba de Kolmogorov - Smirnov, se obtuvieron los siguientes resultados: En las variables «implicación de la alta dirección», «cultura organizativa», «capacidad de gestión», «procesos de gestión del conocimiento» e «indicadores» se obtuvo una $p<.05$, el cual indica que los datos no tienen una distribución normal; sin embargo, para «tecnología», p > 0.05 indica que la hipótesis de normalidad no es rechazada

Debido a los resultados de esta prueba, se procedió a la normalización de los datos mediante el proceso de tipificación a través de la fórmula $Z_{i}=\left(x_{i}-\bar{X}\right)$ $/ \sigma$. En donde $x$ representa el dato a tipificar, $\bar{X}$ significa la media aritmética y $\sigma$, la desviación típica.

\subsection{Modelo de regresión estructural}

En este modelo, se conectan los constructos con los indicadores propuestos para medir cada uno de ellos, de forma que de $x_{1}$ a $x_{7}$ están asociadas a la variable latente independiente $\xi_{1}$, de igual manera, de $x_{8}$ a $x_{15}$ están asociadas a la variable latente independiente $\xi_{2}$, de $x_{16}$ a $x_{21}$ tienen asociación con la tercer variable latente independiente $\xi_{3}$, siguiendo este esquema, de $x_{22}$ a $x_{26}$ están asociadas a la variable latente independiente $\xi_{4}$, y finalmente, de $x_{27}$ a $x_{30}$ están asociadas a la variable latente independiente $\xi_{5}$. Dentro de este contexto, las variables observadas de $y_{1}$ a $y_{4}$ están asociadas a la variable latente dependiente $\eta$. La Tabla 1 muestra esta relación entre las variables latentes, las variables observadas y su descripción.

Tabla 1. Variables observadas que miden cada una de las variables latentes

\begin{tabular}{lrl}
\hline \multicolumn{1}{c}{$\begin{array}{c}\text { Variable } \\
\text { Latente }\end{array}$} & $\begin{array}{c}\text { Variable } \\
\text { observada }\end{array}$ & \multicolumn{1}{c}{ Descripción } \\
\hline $\begin{array}{l}\text { Implicación } \\
\text { de la alta } \\
\text { dirección }\end{array}$ & $\left(x_{1}\right)$ Imp01 & $\begin{array}{l}\text { Conocimiento de política en la empresa para trasmitir } \\
\text { conocimiento }\end{array}$ \\
$\begin{array}{l}\text { (IMPLICGT) } \\
\xi_{1}\end{array}$ & $\left(x_{2}\right)$ Imp02 & $\begin{array}{l}\text { La gestión del conocimiento como aspecto central de la } \\
\text { organización }\end{array}$ \\
& $\left(x_{3}\right)$ Imp03 & $\begin{array}{l}\text { Desarrollo de estrategias para vender el conocimiento } \\
\text { Visitas con otras organizaciones para estudiar experiencias } \\
\text { concretas }\end{array}$ \\
& $\left(x_{5}\right)$ Imp05 & $\begin{array}{l}\text { Asignación de partidas presupuestales } \\
\text { Identificación e impulso periódico de la expertis } \\
\end{array}$ \\
& $\left(x_{7}\right)$ Imp07 & $\begin{array}{l}\text { Reuniones de seguimiento de programas de Gestión del } \\
\text { Conocimiento }\end{array}$ \\
\hline
\end{tabular}




\begin{tabular}{|c|c|c|}
\hline $\begin{array}{l}\text { Variable } \\
\text { Latente }\end{array}$ & $\begin{array}{l}\text { Variable } \\
\text { observada }\end{array}$ & Descripción \\
\hline \multirow{8}{*}{$\begin{array}{l}\text { Cultura para } \\
\text { compartir el } \\
\text { conocimiento } \\
(\text { CULTGT) } \\
\xi_{2}\end{array}$} & $\left(x_{8}\right)$ Cult01 & Existencia de grupos de trabajo interdepartamentales \\
\hline & $\left(x_{9}\right)$ Cult02 & Autonomía y participación en la toma de decisiones \\
\hline & $\left(x_{10}\right)$ Cult03 & Responsabilidad de su propia información \\
\hline & $\left(x_{11}\right)$ Cult04 & Reconocimiento en el desempeño \\
\hline & $\left(x_{12}\right)$ Cult05 & Creación de valor para el cliente \\
\hline & $\left(x_{13}\right)$ Cult06 & Promover el proceso de compartir el conocimiento \\
\hline & $\left(x_{14}\right)$ Cult07 & Clima de trasparencia y confianza \\
\hline & $\left(x_{15}\right)$ Cult08 & Selección, evaluación y compensación por sus contribuciones \\
\hline \multirow{6}{*}{$\begin{array}{l}\text { Capacidad de } \\
\text { Gestión } \\
(\text { CAPGT) } \\
\xi_{3}\end{array}$} & $\left(x_{16}\right)$ Cap01 & Existencia de una persona o un equipo responsable \\
\hline & $\left(x_{17}\right)$ Cap02 & Formación para el equipo de gestión de conocimiento \\
\hline & $\left(x_{18}\right)$ Cap03 & Existencia de un grupo de facilitadores \\
\hline & $\left(x_{19}\right)$ Cap04 & Asistencia a reuniones \\
\hline & $\left(x_{20}\right)$ Cap05 & Presentación de informes periódicos al equipo directivo \\
\hline & $\left(x_{21}\right)$ Cap06 & $\begin{array}{l}\text { Entorno informático específico para la gestión del } \\
\text { conocimiento }\end{array}$ \\
\hline \multirow{5}{*}{$\begin{array}{l}\text { Tecnología } \\
\text { (TECGT) } \\
\xi_{4}\end{array}$} & $\left(x_{22}\right) \operatorname{Tec} 01$ & $\begin{array}{l}\text { Unión de todos los miembros de la organización a través de } \\
\text { la tecnología }\end{array}$ \\
\hline & $\left(x_{23}\right)$ Tec02 & $\begin{array}{l}\text { Memoria organizativa accesible por todos los miembros de la } \\
\text { organización }\end{array}$ \\
\hline & $\left(x_{24}\right) \mathrm{Tec} 03$ & Impulso del uso intensivo de la tecnología \\
\hline & $\left(x_{25}\right) \operatorname{Tec} 04$ & Disponibilidad rápida de la tecnología \\
\hline & $\left(x_{26}\right) \operatorname{Tec} 05$ & Accesibilidad en tiempo real del Sistema de información \\
\hline \multirow{4}{*}{$\begin{array}{l}\text { Indicadores } \\
\text { (INDGT) } \\
\xi_{5}\end{array}$} & $\left(x_{27}\right)$ Ind01 & $\begin{array}{l}\text { Desarrollo de medios para el enlace del conocimiento con } \\
\text { resultados financieros }\end{array}$ \\
\hline & $\left(x_{28}\right)$ Ind02 & Desarrollo de indicadores para gestionar el conocimiento \\
\hline & $\left(x_{29}\right)$ Ind03 & Inventario y valoración del conocimiento en forma periódica \\
\hline & $\left(x_{30}\right)$ Ind04 & Revisión y mejora de indicadores \\
\hline $\begin{array}{l}\text { Procesos } \\
(\text { PROCGT) }\end{array}$ & $\left(y_{1}\right) \operatorname{Proc} 01$ & $\begin{array}{l}\text { Identificación de los déficits de conocimientos y estrategias } \\
\text { correctivas }\end{array}$ \\
\hline \multirow[t]{3}{*}{$\eta$} & $\left(y_{2}\right) \operatorname{Proc} 02$ & $\begin{array}{l}\text { Búsqueda de nuevas ideas por los miembros de la } \\
\text { organización }\end{array}$ \\
\hline & $\left(y_{3}\right) \operatorname{Proc} 03$ & $\begin{array}{l}\text { Formalización del proceso de transferencias de buenas } \\
\text { prácticas }\end{array}$ \\
\hline & $\left(y_{4}\right) \operatorname{Proc} 04$ & Valoración y transferencia del conocimiento tácito \\
\hline
\end{tabular}

Fuente: Elaboración propia con base en la encuesta aplicada. 
Se aplicó el método de estimación de máxima verosimilitud, con una muestra de 135 observaciones y se obtuvieron los coeficientes estimados que se muestran en la Tabla 2. La convergencia se obtuvo a través de 21 iteraciones.

Tabla 2. Coeficientes estimados a través del método de máxima verosimilitud

\begin{tabular}{|c|c|c|c|c|c|c|c|}
\hline $\begin{array}{c}\text { Variable } \\
\text { observada }\end{array}$ & $\begin{array}{l}\text { Coeficiente de } \\
\text { regresión }\end{array}$ & $\begin{array}{c}\text { Error } \\
\text { Estándar } \\
\text { del coef. }\end{array}$ & $\begin{array}{l}\text { Valor } \\
t \text { del } \\
\text { coef. }\end{array}$ & $\begin{array}{l}\text { Error } \\
\text { var }\end{array}$ & $\begin{array}{c}\text { Error } \\
\text { Estándar } \\
\text { de errorvar }\end{array}$ & $\begin{array}{c}\text { Valor } \\
t \text { de } \\
\text { errorvar }\end{array}$ & 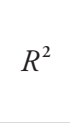 \\
\hline $\operatorname{Imp} 01$ & 68*ImpDirGT & 5) & 8.00 & 0 & & 720 & (1) \\
\hline (2) & 65*ImpDirGT & $(0.080)$ & 8.07 & & (0) & & 0.43 \\
\hline $\operatorname{Imp} 03$ & *ImpDirGT & $(0.08$ & 7.21 & 0.6 & & & 0.36 \\
\hline $\operatorname{Imp} 04$ & $0.81^{*}$ ImpDirGT & $(0.083)$ & 9.80 & 0.49 & & & 0.58 \\
\hline Imp05 & 1*ImpDirGT & $\left(0.0^{\top}\right.$ & 9.02 & 0.4 & & & 51 \\
\hline x6) Imp06 & 58*ImpDirGT & $(0,09-2-2)$ & 6.41 & 0.7 & & 7.67 & 0.30 \\
\hline 7) Imp07 & $0.70^{*} \mathrm{ImpDirGT}$ & $(0.086)$ & 8.11 & 0.6 & & 7.25 & 0.43 \\
\hline x8) Cult01 & $0.43^{*}$ CultGT & $(0.084)$ & 5.15 & 0.77 & $(0.096)$ & 7.98 & 0.20 \\
\hline (x9) Cult02 & $0.42^{*}$ CultGT & $(0.097)$ & 4.36 & 1.07 & $(0.13)$ & 8.04 & 0.14 \\
\hline x10) Cult03 & $0.15^{*}$ CultGT & $(0.083)$ & 1.79 & 0.82 & $(0.100)$ & 8.16 & 0.02 \\
\hline (x11) Cult04 & $72 * C$ T & $.092)$ & 7.94 & 0.77 & & 7.5 & 0.41 \\
\hline & & & & & & & 0.34 \\
\hline & & & & & & & .55 \\
\hline & & & & & & & .51 \\
\hline & & & & & & & 59 \\
\hline & & & & & & & .52 \\
\hline & & & 11.85 & & & & 0.71 \\
\hline (x18) Cap03 & $0.94^{*}$ CapGesGT & $(0.075)$ & 12.46 & 0.29 & & 6.36 & 0.75 \\
\hline (x19) Cap04 & $0.79^{*}$ CapGesGT & $(0.071)$ & 11.06 & 0.34 & & 7.10 & 0.65 \\
\hline (x20) Cap05 & $0.82^{*}$ CapGesGT & $(0.074)$ & 11.18 & 0.36 & & 7.05 & 0.66 \\
\hline (x21) Cap06 & $0.89^{*}$ CapGesGT & $(0.077)$ & 11.61 & 0.36 & $(0.052)$ & 6.87 & 0.69 \\
\hline$(x 22) \operatorname{Tec} 01$ & $0.74^{*} \mathrm{TecGT}$ & $(0.082)$ & 9.04 & 0.51 & & 6.74 & 0.52 \\
\hline$(\mathrm{x} 23) \mathrm{Tec} 02$ & $0.66^{*} \mathrm{TecGT}$ & $(0.074)$ & 9.00 & 0.41 & & 6.76 & 0.51 \\
\hline$(\mathrm{x} 24) \mathrm{Tec} 03$ & $0.67^{*} \mathrm{TecGT}$ & $(0.077)$ & 8.66 & 0.48 & & 6.92 & 0.48 \\
\hline$(\mathrm{x} 25) \mathrm{Tec} 04$ & $0.76^{*}$ TecGT & $(0.082)$ & 9.26 & 0.49 & & 6.63 & 0.54 \\
\hline (x26) Tec05 & $0.75^{*} \mathrm{TecGT}$ & $(0.0)$ & 8.05 & 0.74 & & 7.16 & 0.43 \\
\hline (x27) Ind01 & $0.62^{*} \operatorname{IndGT}$ & $0.081)$ & 7.68 & 0.61 & $(0.080)$ & 7.54 & 0.39 \\
\hline
\end{tabular}




\begin{tabular}{lccccccc}
\hline $\begin{array}{c}\text { Variable } \\
\text { observada }\end{array}$ & $\begin{array}{c}\text { Coeficiente de } \\
\text { regresión }\end{array}$ & $\begin{array}{c}\text { Error } \\
\text { Estándar } \\
\text { del coef. }\end{array}$ & $\begin{array}{c}\text { Valor } \\
t \text { del } \\
\text { coef. }\end{array}$ & $\begin{array}{c}\text { Error } \\
\text { var }\end{array}$ & $\begin{array}{c}\text { Error } \\
\text { Estándar } \\
\text { de errorvar }\end{array}$ & $\begin{array}{c}\text { Valor } \\
t \text { de } \\
\text { errorvar }\end{array}$ & $R^{2}$ \\
\hline (x28) Ind02 & $0.81^{*}$ IndGT & $(0.071)$ & 11.44 & 0.29 & $(0.049)$ & 5.79 & 0.70 \\
(x29) Ind03 & $0.86^{*}$ IndGT & $(0.081)$ & 10.62 & 0.43 & $(0.067)$ & 6.42 & 0.63 \\
(x30) Ind04 & $0.81^{*}$ IndGT & $(0.073)$ & 11.18 & 0.31 & $(0.052)$ & 6.02 & 0.68 \\
(y1) Proc01 & $0.74 *$ ProGT & $(0.080)$ & 9.27 & 0.48 & $(0.072)$ & 6.69 & 0.53 \\
(y2) Proc02 & $0.72 *$ ProGT & $(0.081)$ & 8.95 & 0.51 & $(0.074)$ & 6.85 & 0.51 \\
(y3) Proc03 & $0.70^{*}$ ProGT & $(0.078)$ & 8.98 & 0.47 & $(0.069)$ & 6.84 & 0.51 \\
(y4) Proc04 & $0.63 *$ ProGT & $(0.084)$ & 7.56 & 0.63 & $(0.085)$ & 7.37 & 0.39 \\
\hline
\end{tabular}

Fuente: Elaboración propia con base en datos proporcionados por el Lisrel.

Un modelo de ecuaciones estructurales está compuesto por un modelo estructural y un modelo a la medida. Por tanto, este modelo a la medida queda expresado por las siguientes ecuaciones $x_{1}=\Upsilon_{x 11} \operatorname{ImpDirGT} \xi_{1}+\zeta_{1} ; x_{2}=\Upsilon_{x 2 \text {, }}$ $\operatorname{ImpDirGT} \xi_{2}+\zeta_{2}$ y $x_{3}=\Upsilon_{x 3}$. ImpDirGT $\xi_{3}+\zeta_{3}$. Siguiendo este esquema se muestran las ecuaciones correspondientes en la Tabla 3. Los coeficientes de regresión de este modelo oscilan entre 0.15 de $x_{10}$ y 0.94 de $x_{18}$. La variable de error con el coeficiente más alto está en $x_{9}$ con un valor de 1.07.

Tabla 3. Ecuaciones de regresión del modelo

\begin{tabular}{|c|c|c|}
\hline$x_{1}=0.68^{*} \operatorname{Imp} \operatorname{Dir} G T+0.63$ & $x_{13}=0.83^{*}$ Cult $G T+0.55$ & $x_{25}=0.76^{*} \operatorname{Tec} G T+0.49$ \\
\hline$x_{2}=0.65^{*} \operatorname{Imp} \operatorname{Dir} G T+0.55$ & $x_{14}=0.79 *$ Cult $G T+0.59$ & $x_{26}=0.75^{*} \operatorname{Tec} G T+0.74$ \\
\hline$x_{3}=0.60^{*} \operatorname{ImpDirGT+0.65}$ & $x_{15}=0.93^{*}$ Cult $G T+0.61$ & $x_{27}=0.62 * \operatorname{Ind} G T+0.61$ \\
\hline$x_{4}=0.81^{*} \operatorname{ImpDir} G T+0.49$ & $x_{16}=0.78^{*}$ CapGesGT+0.57 & $x_{28}=0.81^{*} \operatorname{Ind} G T+0.29$ \\
\hline$x_{5}=0.71^{*} \operatorname{ImpDirGT}+0.48$ & $x_{17}=0.90^{*}$ CapGes $G T+0.34$ & $x_{29}=0.86^{*} \operatorname{In} d G T+0.43$ \\
\hline$x_{6}=0.58^{*} \operatorname{Imp} \operatorname{Dir} G T+0.79$ & $x_{18}=0.94^{*}$ CapGes $G T+0.29$ & $x_{30}=0.81^{*} \operatorname{Ind} G T+0.31$ \\
\hline$x_{7}=0.70^{*} \operatorname{Imp} \operatorname{Dir} G T+0.64$ & $x_{19}=0.79^{*}$ CapGes $G T+0.34$ & $y_{1}=0.74^{*} \operatorname{Pro} G T+0.48$ \\
\hline$x_{8}=0.43^{*}$ Cult $G T+0.77$ & $x_{20}=0.82^{*}$ CapGes $G T+0.36$ & $y_{2}=0.72 * \operatorname{Pro} G T+0.51$ \\
\hline$x_{9}=0.42^{*}$ Cult $G T+1.07$ & $x_{21}=0.89^{*}$ CapGesGT 0.36 & $y_{3}=0.70 * \operatorname{Pro} G T+0.47$ \\
\hline$x_{10}=0.15^{*}$ Cult $G T+0.82$ & $x_{22}=0.74^{*} \operatorname{Tec} G T+0.51$ & $y_{4}=0.63^{*} \operatorname{Pro} G T+0.63$ \\
\hline$x_{11}=0.73^{*}$ Cult $G T+0.77$ & $x_{23}=0.66^{*} \operatorname{Tec} G T+0.41$ & \\
\hline$x_{12}=0.61^{*}$ Cult $G T+0.73$ & $x_{24}=0.67^{*} \operatorname{Tec} G T+0.48$ & \\
\hline
\end{tabular}

Fuente: Elaboración propia con base en los resultados del software Lisrel. 
Se calcularon los coeficientes de correlación de las variables: Implicación de la alta dirección, cultura organizativa, capacidad de gestión, tecnología, procesos para gestionar el conocimiento e indicadores, analizadas en esta investigación (Tabla 4). La mayor intensidad en relación se obtiene entre la cultura organizativa con otras variables evaluadas; por ejemplo, "cultura organizativa con procesos de gestión del conocimiento» obtuvo un valor de 0.86 , «cultura organizativa con el uso de tecnología» obtuvo 0.77 , «cultura organizativa con la participación de la alta dirección el coeficiente de correlación» fue de 0.75 , "cultura organizativa con capacidad de gestión el valor de correlación" fue de igual forma 0.75 . Otra correlación muy significativa está entre los indicadores con los procesos de gestión del conocimiento con un coeficiente de 0.76. Por otro lado, se observó que la capacidad de gestión con la tecnología tiene una correlación baja, pero, en general, existe una gran correlación entre todas las escalas analizadas.

Tabla 4. Matriz de correlación

\begin{tabular}{lccccc}
\hline & $\begin{array}{c}\text { Participación de } \\
\text { la Alta Dirección } \\
\text { (IMPLICGT) }\end{array}$ & $\begin{array}{c}\text { Cultura } \\
\text { organizativa } \\
\text { (CULTGT) }\end{array}$ & $\begin{array}{c}\text { Capacidad } \\
\text { de gestión } \\
\text { (CAPGT) }\end{array}$ & $\begin{array}{c}\text { Tecnología } \\
\text { (TECGT) }\end{array}$ & $\begin{array}{c}\text { Procesos de } \\
\text { gestión del } \\
\text { conocimiento } \\
\text { (PROCGT) }\end{array}$ \\
\hline CULTGT & 0.75 & & & & \\
CAPGT & 0.64 & 0.75 & & & \\
TECGT & 0.58 & 0.77 & 0.49 & & \\
PROCGT & 0.62 & 0.86 & 0.68 & 0.70 & 0.76 \\
INDGT & 0.58 & 0.68 & 0.71 & 0.53 & \\
\hline
\end{tabular}

Fuente: Elaboración propia con base en los resultados del software Lisrel.

En el modelo de regresión estructural de la Figura 2, se muestran los resultados de la correlación entre las variables latentes que se han descrito en la Tabla 4. Existe una flecha bidireccional entre participación de la alta dirección (IMPLICGT) y cultura organizativa CULTGT con coeficiente de correlación de un valor de 0.75 , para las variables latentes IMPLICGT y CAPGT tiene la correlación de 0.64 , asimismo, para las variables IMPLICGT y TECGT su correlación es de 0.58, entre IMPLICGT y PROCGT su coeficiente de correlación es de 0.62, y dentro de implicación de la dirección (IMPLICGT) y los indicadores de gestión del conocimiento (INDGT), el coeficiente de correlación es de 0.58 . Bajo este mismo esquema, se presenta la correlación de CULTGT, CAPGT, TECGT, PROCGT e INDGT con las otras variables 
latentes del modelo. De las variables latentes salen flechas hacia las variables observadas indicando el coeficiente de regresión mostrados (ver Tabla 4) para esa variable, de forma que, de la variable latente INDGT hacia la variable observada $\left(x_{1}\right)$ IMP01, el coeficiente es de 0.68 . La variable error se encuentra representada con la flecha que entra a la izquierda de la variable $\left(x_{1}\right)$ IMP01 con un valor de 0.63.

Figura 2. Modelo de regresión estructural para los factores generadores de éxito

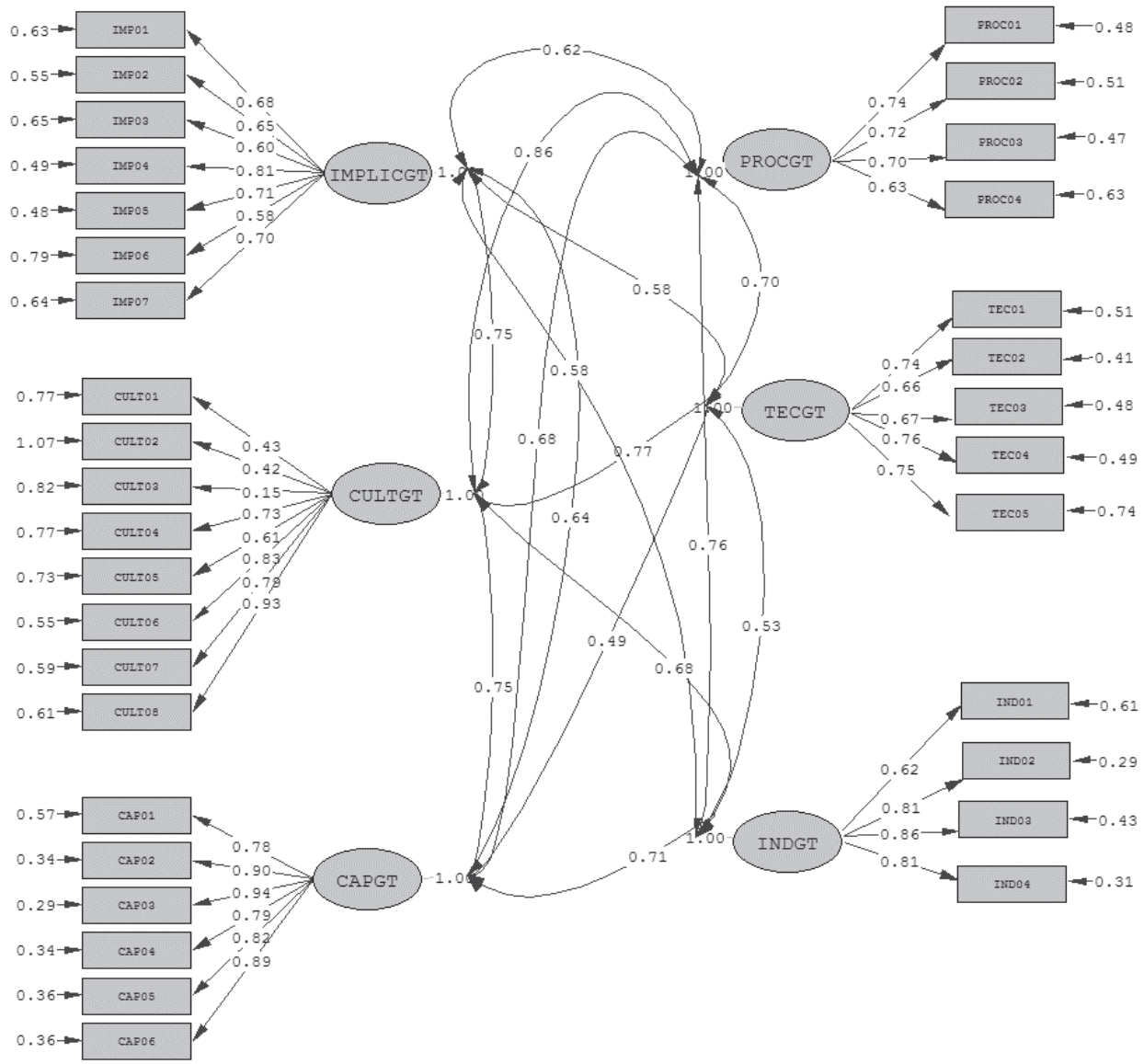

Chi-Square $=875.96, d f=512, \mathrm{P}$-value $=0.00000, \mathrm{RMSEA}=0.073$

Fuente: Elaboración propia con base en apoyo del software Lisrel. 
En el modelo presentado (ver Figura 2), se tiene un total de 34 variables endógenas $(s=34)$, de forma que el número no redundante de la matriz $\Sigma$ es de $s(s+1) / 2=595$. En los índices de ajuste del modelo, se obtuvo una Chi-cuadrada $\left(\chi^{2}\right)$ de 875.96 con 512 grados de libertad (gl) y p $<0.001$. El parámetro $-2 \ln (L)$, para el modelo saturado, es de 10244.36 e indica que el modelo es suficientemente parametrizado, porque cada punto de datos se abarca de manera positiva, mientras que el $-2 \ln (L)$ para modelo ajustado es de 11168.77. El error cuadrático medio de aproximación (RMSEA) es de 0.073. El intervalo de confianza al 90\% (IC), para el RMSEA, es de 0.065 y 0.081; el índice de ajuste normado (NFI: normed fit index), 0.9; índice de ajuste no normado (NNFI: non-normed fit index), 0.95; índice de ajuste parsimonioso normado (PNFI: parsimony normed fit index), 0.83; índice de ajuste comparativo (CFI: comparative fit index), 0.96; índice de ajuste incremental (IFI: incremental fit index), 0.96, y el índice de ajuste relativo (RFI: relative fit index), 0.90. Asimismo, la raíz media cuadrática residual (RMR: root mean square residual) es de 0.073; índice RMR estandarizado, 0.069; el índice de bondad y ajuste (GFI: goodness of fit index), 0.72; finalmente, el índice corregido de bondad y ajuste (AGFI: adjusted goodness of fit index), 0.68. Los resultados de los indicadores concluyen que el modelo propuesto es adecuado.

Una vez que se obtuvo la matriz de correlación, se obtuvo la matriz de covarianza para obtener la ecuación de regresión lineal múltiple, la cual se estableció a través de la fórmula general $Y_{t}=\beta_{0}+\beta_{1} X_{1}+\beta_{2} X_{2}+\beta_{3} X_{3} \ldots+\beta_{n}$ $X_{n}+\epsilon$. En esta fórmula, $Y_{t}$ representa la variable dependiente, mientras que $X_{1}, X_{2}, X_{3} \ldots X_{n}$, representan las variables independientes. $\beta_{0}, \beta_{1}, \beta_{2} \ldots \beta_{n}$ miden la influencia que tienen las variables explicadas. $\epsilon$ representa la perturbación aleatoria que acumulan los factores de la realidad no controlables u observables y que se asocian con el azar. En esta investigación, $Y_{t}$ representa los procesos de gestión del conocimiento, $X_{1}$, la participación de la alta dirección, $X_{2}$, la cultura organizacional, $X_{3}$, la capacidad de gestión, $X_{4}$, la influencia de la tecnología, finalmente, $X_{5}$ representa el uso de indicadores. En los resultados de la regresión múltiple, el valor de la constante $\beta_{0}$ no tiene ningún impacto porque generó un valor de cero. Error $z$ representado por $\epsilon$ obtuvo un valor de 0.019 .

La ecuación de regresión lineal múltiple muestra que, para los procesos de gestión del conocimiento, la cultura de la organización tiene un impacto determinante de .68; el impacto de los indicadores es de .36; de la tecnología, .077 , mientras que para la capacidad de gestión es de .052 . En los procesos de gestión del conocimiento, se observa que la participación de la alta dirección 
en estos procesos no tiene una participación significativa, ya que su coeficiente es de -.11. El valor de error $z$ es de .019 e indica que existen otros elementos que intervienen en los procesos de gestión del conocimiento.

Ecuación de regresión lineal múltiple:

$$
\begin{gathered}
\mathrm{Y}_{\mathrm{PROCGT}}=-0.11^{*} X_{\text {IMPLICGT }}+0.68^{*} \mathrm{X}_{\text {CULTGT }}-0.052^{*} \mathrm{X}_{\text {CAPGT }}+0.077^{*} \mathrm{X}_{\text {TECGT }} \\
+0.36 * \mathrm{X}_{\text {INDGT }}+0.019
\end{gathered}
$$

\section{RESULTADOS Y DisCUSIÓN}

Se analizó individualmente cada una de las variables (implicación de la alta dirección, cultura organizativa, capacidad de gestión, tecnología, procesos de gestión del conocimiento e indicadores) tomando como base el modelo propuesto por Molina y Marsal (2002). Después se realizó una correlación para identificar el grado de asociación entre las variables, midiendo la fuerza y la dirección entre ellas; también se elaboró la regresión lineal entre todas las variables para identificar cómo influyen en los procesos de gestión del conocimiento, explorando y cuantificando su impacto.

\subsection{Participación de la alta dirección en la gestión del conocimiento}

En esta dimensión, se consideró importante si las personas tenían conocimiento de las políticas en la institución para transmitir el conocimiento, si suponían que la gestión del conocimiento era un aspecto central, además si se desarrollan estrategias para transferir el conocimiento. Se reflexionó también la intervención de los directivos para integrar conocimientos desde experiencias concretas en organizaciones ajenas, si creen que la institución se preocupa por gestionar el conocimiento previendo partidas presupuestales a través de programas definidos, si se identifican y se impulsa la expertis o las competencias clave de los trabajadores y si hay reuniones contantes para dar seguimiento a los programas de gestión del conocimiento. Por ello, para la dimensión de la participación de los directivos en la gestión del conocimiento, se observó que la mayoría de las personas están de acuerdo en que los directivos se integran y apoyan la gestión del conocimiento; sin embargo, muchos desconocen si se previenen partidas presupuestales, si es una política de la empresa y si se realizan reuniones para gestionar el conocimiento.

En los resultados obtenidos, se observó que el 38\% de los encuestados están de acuerdo en que existen políticas para transmitir el conocimiento en la institución, el 43\% considera que gestionar el conocimiento es un aspecto central en la institución, el 42\% está consciente de que se desarrollan estrate- 
gias para vender el conocimiento en el mercado, el 38\% comenta que se han realizado visitas con otras organizaciones para estudiar experiencias concretas; sin embargo, el 37\% no sabe si se han previsto partidas presupuestales para apoyar la transmisión del conocimiento. El 42\% considera que la institución identifica e impulsa periódicamente su expertis o competencias clave, pero el $36 \%$ no sabe si se celebran reuniones para dar seguimiento a los programas de gestión del conocimiento.

\subsection{Cultura organizacional}

En esta dimensión, se identifica si existen grupos interdepartamentales para gestionar el conocimiento, si se tiene autonomía y participación en la toma de decisiones, si los trabajadores son responsables de su propia información, si los mecanismos de reconocimiento de la excelencia en el desempeño impulsan la cultura de la organización; también fue relevante analizar si el trabajador reconoce que gestionar el conocimiento impulsa la creación de valor para el cliente; así como la organización anima y facilita el proceso de compartir el conocimiento sobre el clima de transparencia y confianza que caracteriza a la institución, y si las personas se seleccionan, se evalúan y son compensadas por su contribución al desarrollo.

De acuerdo con los resultados, se observó que el $44 \%$ percibe que existen grupos interdepartamentales para gestionar el conocimiento, el 33\% coincide en que los empleados tienen alta autonomía en la toma de decisiones; sin embargo un $27 \%$ está en desacuerdo señalando que no tienen libertad en la toma de decisiones, el $47 \%$ se considera responsable de su propia información, el 37\% piensa que existen mecanismos de reconocimiento de excelencia en el desempeño de su trabajo, el $41 \%$ reconoce que el objetivo de la gestión del conocimiento es crear valor para el cliente, el $37 \%$ cree que la organización anima y facilita la gestión del conocimiento, el 33\% señala que en la institución existe un clima de transparencia y confianza, finalmente, se observó que el 30\% ignora si la selección de personas, la evaluación y la compensación contribuye con el desarrollo de la institución.

\subsection{Capacidad de gestión}

En la capacidad de gestión, se observó que el 36\% no sabe si existe una persona o un equipo responsable de la gestión del conocimiento, asimismo, el $40 \%$ desconoce si el equipo de gestión del conocimiento recibe o ha recibido una formación específica, el $41 \%$ no tiene idea si existe un grupo facilitador para gestionar el conocimiento, el 39\% ignora si participan en reuniones, el 
$44 \%$ no tiene conocimiento si el equipo de gestión del conocimiento presenta informes periódicos a directivos, finalmente, el 39\% desconoce si existe un entorno informático específico del conocimiento administrado por un equipo responsable. En general, se observó que la mayoría ignora si en la institución existe un grupo responsable de gestionar el conocimiento.

\subsection{Tecnología}

En esta dimensión, se analizó la forma en su uso e impulsa la gestión del conocimiento uniendo a los miembros de la organización y a los clientes, proveedores y socios. Asimismo, se observó que el 50\% está de acuerdo en que esta herramienta es un enlace entre todos los integrantes tanto internos como externos. En la disponibilidad de información, el $47 \%$ considera que se ha creado una memoria organizativa disponible para todos. El $44 \%$ considera que la institución promueve el uso de la tecnología de los empleados. El $41 \%$ está de acuerdo en que la institución pone a su disposición la tecnología que facilita el trabajo en equipo. En cuanto al acceso a la información en tiempo real, el 36\% está de acuerdo que el sistema es accesible e inteligente, mientras el $24 \%$ está totalmente de acuerdo con este aspecto. En general, se observó que la mayoría percibe y está de acuerdo en que es una herramienta que impulsa la gestión del conocimiento.

\subsection{Procesos de gestión del conocimiento}

Para analizar los procesos de gestión del conocimiento, se observó que el 40\% desconoce si se han identificado los déficits del conocimiento y si se han tomado acciones para superar los contratiempos. El 43\% está de acuerdo en que todos los integrantes de la organización están implicados en buscar nuevas ideas y que la organización ha formalizado el proceso de transferir buenas prácticas. Por su parte, el 34\% aprueba que la institución valora y transfiere el conocimiento tácito de sus trabajadores. Asimismo, se observó la tendencia en los resultados en donde la mayoría está de acuerdo en que los directivos impulsan la gestión del conocimiento a través de la búsqueda de ciertas ideas, de la transferencia de las buenas prácticas y de valorar el conocimiento tácito.

\subsection{Indicadores}

Se analizó si se han desarrollado estrategias para vincular el conocimiento con resultados financieros, se observó que el 37\% está «ni de acuerdo ni en desacuerdo", mientras que el $33 \%$ está de acuerdo que existe ese enlace, el $42 \%$ no sabe de la existencia de indicadores. El $34 \%$ no sabe si se tiene 
un inventario del conocimiento que sea valorado periódicamente, el $47 \%$ ignora si los indicadores de conocimiento son revisados y valorados constantemente. En resumen, la mayoría de las personas desconoce los factores analizados para esta variable, aunque la tendencia en las personas muestra que están de acuerdo en que existen indicadores para gestionar el conocimiento y que se les da seguimiento periódicamente.

\section{CONCLUSIONES}

De acuerdo con los resultados, se observó que la alta dirección de la institución se involucra y busca estrategias para impulsar el conocimiento adoptando políticas para transmitir el conocimiento, visitando a otras instituciones con la finalidad de analizar experiencias concretas y asignando partidas específicas para impulsar la gestión del conocimiento. La cultura de la organización fue un factor muy importante para los procesos de gestión del conocimiento, debido a que existen grupos de trabajo interdisciplinarios, tienen alta autonomía y participación en la toma de decisiones, existe responsabilidad sobre la información que manejan, se reconoce la excelencia en el desempeńo, se fortalece y se impulsa el proceso de gestión del conocimiento. En cuanto a la capacidad de gestión, se manifiesta desconocimiento sobre la existencia de grupos de apoyo. Se observó que la tecnología es una herramienta que tiene un fuerte impacto en la gestión del conocimiento que cuenta con herramientas tecnológicas y estrategias adecuadas para distribuir la información entre todos los integrantes. En los indicadores, se desconoce si se toman acciones para corregir algún déficit en la gestión del conocimiento, pero todos los integrantes están implicados en buscar ideas nuevas, la formalización de buenas prácticas y la valoración del conocimiento tácito

Para responder la pregunta de investigación "¿Qué factores impulsan la gestión del conocimiento en una Institución de Educación Superior?», se puede concluir que se establecen estrategias basadas en la cultura de la organización, por tanto, la implementación de indicadores y el uso de la tecnología impulsan la gestión del conocimiento. Tomando como base que la cultura organizacional tiene una relación positiva con otras variables, se puede inducir que el clima de la organización es propicio para gestionar el conocimiento. Aunque no se tiene un equipo responsable, los procesos de gestión del conocimiento se llevan a cabo a través de estrategias para motivar la innovación constante, buscando ideas nuevas y formalizando los procesos de documentar las buenas prácticas. Por ello, se propone la existencia de un equipo de gestión del conocimiento que sea responsable de las actividades inherentes a este proceso y que la gestión del conocimiento sea parte central; por tanto, se reco- 
mienda también asignar partidas presupuestales para crear un equipo que sea parte de la estructura orgánica institucional para promover las competencias de los trabajadores.

\section{REFERENCIAS BIBLIOGRÁFICAS}

Argote, L. (1999). Organizational learning: creating, retaining and transferring knowledge. Boston, US: Kluwer Academic Publishers.

Byrne, B. M. (2010). Multivariate applications series. Structural equation modeling with amos: basic concepts, applications, and programming. (2nd. ed.). Nueva York, US: Routledge/Taylor \& Francis Group.

Cabero, A. J., Ballesteros, R. C. y López M. E. (2015). Los mapas conceptuales interactivos como recursos didácticos en el ámbito universitario. Revista Complutense de Educación, 26, 15-31. https://doi.org/10.5209/rev_ RCED.2015.v26.43791

De Freitas, V. y Yaber, G. (2015). Una taxonomía de los factores clave de éxito en la implantación de sistemas de gestión del conocimiento en instituciones de educación superior. GECONTEC: Revista Internacional de Gestión del Conocimiento y la Tecnología, 3(1), 69-86.

Digman, L. A. (1990). Strategic management: concepts, decisions, cases. (2nd. Ed.). Boston, US: Homewood, Ill.: BPI/Irwin.

Fernández, M. M. (2015). Los portales del conocimiento en las organizaciones contemporáneas. e-Ciencias de la Información, 5(1), 1-18. doi: https://doi. org/10.15517/eci.v5i1.17036

Grace, J. B. (2006). Structural equation modeling and natural systems. Nueva York, US: Cambridge University Press. https://doi.org/10.1017/ CBO9780511617799

Gujarati, N. D. y Porter, C. D. (2009). Econometría. (5a. ed.). México, D. F.: McGraw-Hill.

Gutiérrez-Doña, B. (2008). Cuaderno metodológico 2: modelos lineales estructurales: conceptos básicos, aplicaciones y programación con LISREL. San José, CR.: Instituto de Investigaciones Psicológicas.

Hartwick, J. y Barki, H. (2001). Interpersonal conflict and its management in information system development. MIS Quarterly, 25(2), 195-228. https:// doi.org/10.2307/3250929

Herrera, C. J. F. y Giraldo, V. O. L. (2010). Apropiación de conocimiento entre instituciones privadas de educación: transferencia del proyecto cup2 de uniandes a uniminuto. Journal of Information Systems and Technology Management. 7(1), 33-70. https://doi.org/10.4301/S1807-17752010000100002 
Kline, B. R. (2011). Principles and practice of structural equation modeling. (3ra. ed.). Nueva York, US: The Guilfors Press.

KnowledgeMaster.(2007).ElpuestodetrabajodelGestordelConocimiento. Recuperado de http://conceptmaps.it/KM-KnowledgeManagerJob-esp.htm

Ko, D. G., Kirsch, L. J. y King, W. R. (2005). Antecedents of knowledge transfer from consultants to clients in enterprise system implementations. Management Information System Quarterly, 29(1), 59-85. https://doi. org/10.2307/25148668

López, D., Marulanda, C. E. y López M. (2015). Métricas de valoración de la gestión del conocimiento para las pequeńas y medianas empresas del sector tecnologías de información en el triángulo del café en colombia. Información Tecnológica, 26(3), 173-183. https://doi.org/10.4067/ S0718-07642015000300020

Manzano, P. A. y Zamora, M. S. (2009). Sistema de ecuaciones estructurales: una herramienta de investigación. Cuaderno técnico 4. México: Centro Nacional de Evaluación para la Educación Superior, A.C. (Ceneval).

Molina, J. L. y Marsal, S. M. (2002). La gestión del conocimiento en las organizaciones. Colección de Negocios, Empresa y Economía. Libros en red.

Schumacker, R. E. y Lomax, R. G. (2010). A beginner's guide to structural equation modeling (3a. ed.). Nueva York, US: Taylor and Francis Group, LLC.

Secretaría de Educación Pública (2015). Transferencia de conocimientos $y$ tecnología. Recuperado de http://dgeta.sems.gob.mx/es/dgeta/ transferencia_tecnologica1

Sik-Yum, L. (2007). Structural equation modeling. A bayesian approach. Chichester, England: John Wiley \& Sons Ltd.

Ullman, B. J. (2006). Structural equation modeling: reviewing the basics and moving forward. Journal of Personality Assessment, 87(1), 35-50. https:// doi.org/10.1207/s15327752jpa8701_03 